\title{
A GENERIC AUGMENTED REALITY TELESCOPE FOR HERITAGE VALORIZATION
}

\author{
S. Chendeb ${ }^{\mathrm{a}, *}$, T. Ridene ${ }^{\mathrm{a}, *}$, L. Leroy ${ }^{\mathrm{a}, *}$ \\ ${ }^{a}$ CITU PARAGRAPHE, Paris 8 University, 2 rue de la Liberté 93526 Saint-Denis Cedex, Paris region, France - \\ (safwan.chendeb, taha.ridene, laure.leroy)@citu.fr
}

Commission II, WG II/2

KEY WORDS: Augmented Reality, Virtual Reality; Telescope; Heritage Valorisation.

\begin{abstract}
:
Heritage valorisation is one of the greatest challenges that face countries in preserving their own identity from the globalization process. One of those scientific areas which allow this valorisation to be more attractive and at its bravest is the augmented reality. In this paper, we present an innovative augmented reality telescope used by tourists to explore a panoramic view with optional zooming facility, allowing thereby an accurate access to heritage information. The telescope we produced is generic, ergonomic, extensible, and modular by nature. It is designed to be conveniently set up anywhere in the world. We improve the practical use of our system by testing it right in the heart of Paris within a specific use case.
\end{abstract}

\section{INTRODUCTION}

The use of virtual reality and augmented reality is a matter of increasing interest. Several areas are affected by and benefits from the improvements in these two scientific domains. Through them the production of many applications became all of a sudden possible and a lot of business models now integrate with success virtual reality and augmented reality into building products. We can cite as applications: parachuting simulation used in military context [1]; surgery in health care $[2,3,4]$; analysis of sports performance [5]; astronomy teaching in science and education context $[6,20,21]$; etc. For further details about virtual reality and augmented reality applications surveys please refer to $[7,8,9]$.

The work described in this paper is related to the TerraNumerica whose aim is to generate realistic and georeferenced 3D maps of urban areas around Paris and to use them for heritage valorisation purposes. In this study we present an innovative augmented reality Telescope. Placed on the top of specific monuments, this platform makes it possible for tourists to discover a panoramic view available in the area covered by the Telescope, and to provide access to accurate information about specific monuments and heritage information (Fig. 1).

The main contributions to this paper can be identified as follows. Firstly, we explain how and why our system can be regarded as generic, ergonomic, extensible, and modular. Secondly, we present the nuts and bolts of the production process of this kind of platform (including software, electronic, and hardware), this time from a very practical point of view. Thirdly, we show how to elaborate a testing protocol in a real use case for this kind of augmented reality applied to heritage valorisation.

This paper is organized as follows. Section II presents a state of the art augmented reality application focusing on heritage valorisation. Section III presents the strengths of our Telescope. Section IV reveals the particulars of our system. Section V presents our testing protocol and a practical use case. We finally deliver our conclusions and prospects for future works.

\section{AUGMENTED REALITY FOR HERITAGE VALORIZATION}

In the context of Heritage Valorisation different approaches based on augmented reality were proposed in previous works. One can for example provide a basic classification by separating indoor and outdoor environments. In this case we propose a categorization relying on two concepts:

- A dedicated application using a mobile device on a specific site. The mobile device can be a smart phone, a dedicated device but also augmented reality glasses (Google Glass ${ }^{1}$, Laster Glass ${ }^{2}$ ). In this category we can cite applications for museology $[10,11,12]$, archaeology $[13,14]$, specific historical site visiting as Versailles Castle, ${ }^{3}$, etc.

- A dedicated application using a fixed device on specific site. The fixed device usually comprises an immersive window $[17,18,23]$ and is installed on a rigid platform. It is a kind of device which allows a point of view of the immersion type which basically consists in moving the screen and getting to see what lies behind it, optionally through a virtual improving technique. In this category we can mainly cite the Telescopes. In this case of immersive windows, the head of the subject is pressed to the screen, in order to produce a concentric immersive point of view (the movements of the head and the movement of the camera are equal). They often are designed in the same fashion as traditional telescopes. Our system belongs to this category. TABLE I. includes a list of existing Telescopes designed for heritage valorisation applications. In the next section we will present the strengths of our system.

\footnotetext{
${ }^{1}$ Google Glass : https://plus.google.com/+projectglass/

${ }^{2}$ Laster Technologie : http://www.laster.fr/

${ }^{3}$ Chateaux de Versailles : www.gvn.chateauversailles.fr
} 

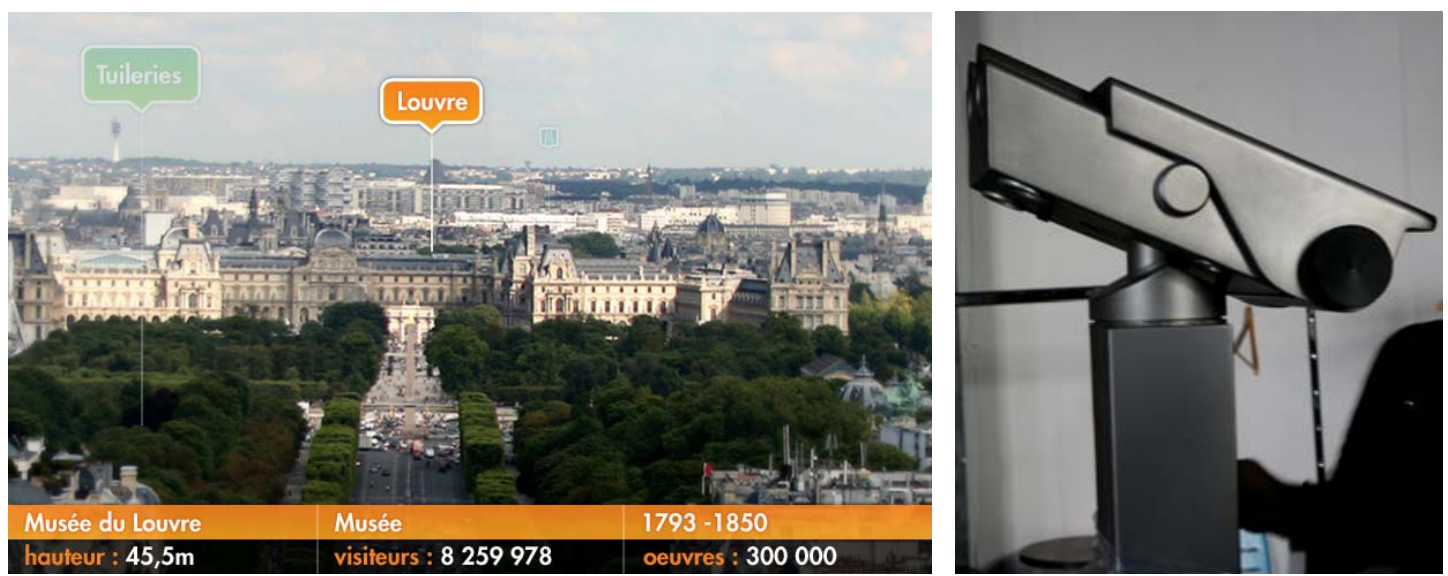

Fig. 1. Augmented Reality Telescope for Heritage Valorization. (left) "Paris, Champs Elysée" avenue panoramic view with a focus on "Palais du Louvre". (right) Our Telscopoe.

TABLE I. EXISTING TELESCOPE FOR HERITAGE VALORIZATION PURPOSES

\begin{tabular}{|l|l|l|}
\hline Team & Application & Ref \\
\hline $\begin{array}{l}\text { Beijing Inst. of } \\
\text { Technol., Beijing, } \\
\text { China }\end{array}$ & $\begin{array}{l}\text { Digital reconstruction of } \\
\text { Yuangmingyuan in Beijing China }\end{array}$ & {$[15]$} \\
\hline $\begin{array}{l}\text { Asociación } \\
\text { VICOMTech, San } \\
\text { Sebastian, Spain }\end{array}$ & $\begin{array}{l}\text { Enhancing Cultural Tourism } \\
\text { DonostiaSan Sebastian, Spain. }\end{array}$ & {$[16]$} \\
\hline $\begin{array}{l}\text { Fraunhofer Institute for } \\
\text { Computer Graphics, } \\
\text { Fraunhofer, Germany }\end{array}$ & The augmented reality ocular & {$[21$,} \\
\hline $\begin{array}{l}\text { The University of } \\
\text { Nottingham, } \\
\text { Nottingham, UK }\end{array}$ & $\begin{array}{l}\text { A mixed reality tripod for outdoor } \\
\text { to replace historical characters } \\
\text { (more an immersive windows than } \\
\text { a telescope) }\end{array}$ & {$[23]$} \\
\hline
\end{tabular}

\section{THE STRENGHTS OF OUR SYSTEM}

\subsection{Generic System}

Our system is as generic as possible. It relies upon a dynamic calibration process with a localization module. Our system is easy to move from one site to another, and it is so designed that it can at the same time be regarded as belonging to the category of fixed devices for augmented reality applications for heritage valorisation purposes, but with the added value that its included dynamic functionalities make it perfectly suitable to be mobile or duplicated all over the world, as well as to other purposes in dedicated augmented reality contexts (touristic, etc). As a result, the telescope can be set up on every site around the world: l'Arc de Triomphe (Paris, France), Liberty Island (New-York, USA), El Corcovado (Rio de Janeiro, Brazil)... to move it around, we simply need to integrate the required augmented reality information and to specify the calibration coordinates.

\subsection{Open Software System}

Our system includes a modular software architecture which not only allows for further developments on the applications tested on it, but also a seamless adaptability to numerous fields of application besides heritage valorisation. Furthermore, considering the large number of existing and potential industrial software modellers, we chose to design the core of our software architecture for the highest portability and with a view to a straightforward interfacing to all kinds of 3D modellers: we have so far conducted successful tests with Virtools ${ }^{4}$, 3DVia Studio ${ }^{5}$, OpenSceneGraph ${ }^{6}$.

\subsection{Ergonomic System}

\subsubsection{Vision}

\subsubsection{Stereoscopic- monoscopic vision}

We chose to avoid stereoscopic vision, on the simple reasoning that our device is meant to appeal to the widest audience, especially children. Stereoscopic vision is known to generate eye strain [29], and in some cases possibly eye trauma, particularly where children are concerned. Moreover, with respect to very distant buildings, stereoscopic vision cannot improve relief perception [24]. So our preference went to a monoscopic vision instead of a stereoscopic one.

\subsubsection{Accommodation}

Accommodation breaks can prove to be a strain on the eyes [25]. Keeping this in mind we preferred images of building and information balloons to be in the same accommodation plane. Therefore we decided to make a film of the landscape and to insert information balloons in the same plane. This is the advantage of the video see-through.

\subsubsection{Design}

\subsubsection{Affordance}

We wanted the use of our telescope to be easily and readily understood by the public at large, and quality of affordance was consequently of paramount importance to us.

\subsubsection{Security}

Nobody wants to be responsible for causing injuries to people's hands or other bodily parts, especially children. But we are in non surveyed public places, so we decided not to tempt fate and have holes present in our device where hands might get caught in.

\footnotetext{
${ }^{4}$ Virtools : www.virtools.com/

${ }^{5}$ 3DVIA Studio : http://www.3dvia.com/

${ }^{6}$ OpenScenegraph : http://www.openscenegraph.org/projects/osg
} 


\subsubsection{Counterbalancing}

Telescopes can prove to be heavy and at times awkward to handle and we wanted its movements and weight to be counterbalanced. Moreover, it seemed desirable to keep down the velocity of its movements hydraulically.

\section{DETAILS OF OUR SYSTEM}

\subsection{Mechanical Architecture}

Our aim was to produce a telescope presenting a very good affordance, and we accordingly elected to design it in a most traditional fashion.

First of all, we chose a design very much corresponding to that of a customary telescope (Fig. 2).
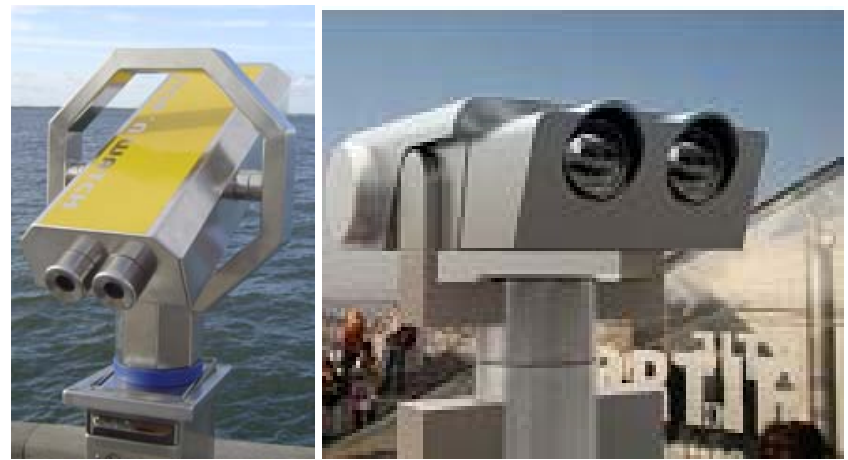

Fig. 2. (left) Traditional toursitic Telescope. (right) First design of the Telescope.

But we were concerned that people, especially children, would get their hands caught in the cavity between the axis and the telescope itself. So we have slightly altered the design to take care of this issue (Fig. 3).

\subsection{Interface architecture}

Our new design included a microphone and headphones to make future developments possible. The whole of the telescope is, needless to say, waterproof.

The angles are measured by an absolute coder, and the camera has a variable field of view. The vision is ensured by a micro display device.

Focus-command and selective interface are provided by toothed wheel (Fig. 3).

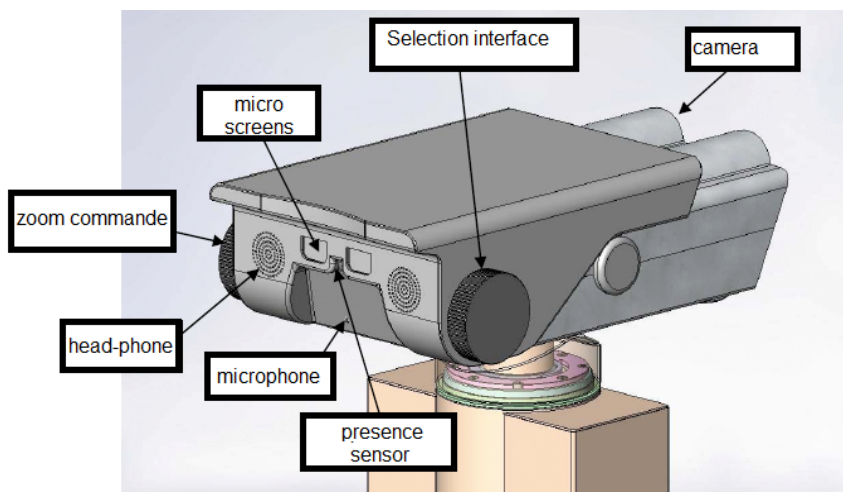

Fig. 3. Mecanical Architecture of the Telescope.

\subsection{Hardware Architecture}

The acquisition card has been so designed as to allow for many connection schemes (Fig. 4). It includes three absolute coders, six incremental coders, two thermometers, a light sensor, two serial ports for camera control, USB, Ethernet ports, and digital/analog input and output. The card comprises 3 CPU's: one master and to slaves for the various connections.

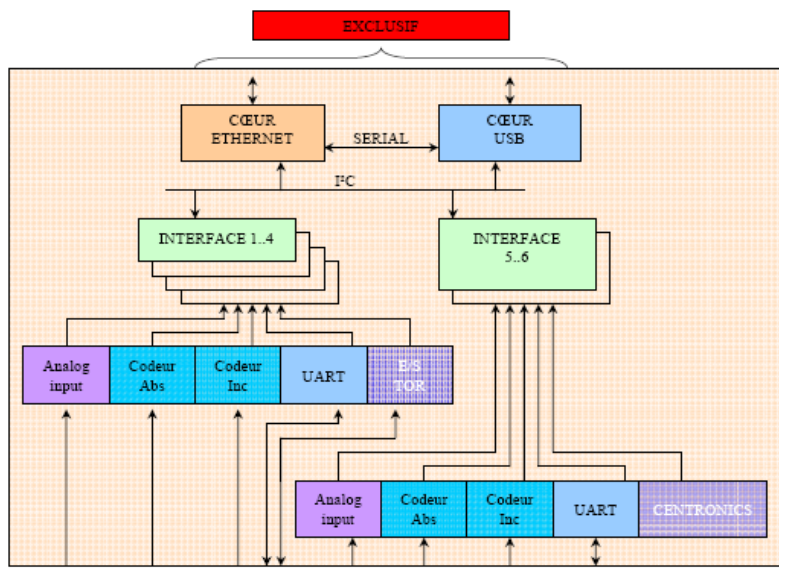

Fig. 4. Electronic shema architecture. General organization and interfaces between the different components.

\subsection{Software Architecture}

We also wanted the telescope to be as modular as possible. So we introduced an abstraction layer: $V R P N^{7}$ (Virtual Reality Peripheral Network) server. $V R P N$ is a free library which allows for a transparent interface between an application (on a computer) and a hardware device (linked to another computer on the network). This library relies on a client-server architecture: the server unit consists of a computer which collects data from the coders and the camera, and the client unit consists of computer whose task is to compute the application.

This type of library has numerous advantages:

- Network abstraction;

- Execution speed;

- Code portability;

- Compatibility with many virtual reality softwares (included Virtools);

- Independent Tracking;

- Asynchronous Non-blocking communications.

The augmented reality engine is based upon an open-source library such as OpenScenegraph for $3 \mathrm{D}$ rendering, ARToolkit ${ }^{8}$ for virtual objet insertion; it is connected to a $3 \mathrm{D}$ database (produced by the partners of the project TerraNumerica) which contains 3D models of Paris buildings. There are other available peripheral databases providing static information (for example historical) or dynamic (for example weather forecasts or observations). The Error! Reference source not found. includes a global schema about our Telescope software layers.

\footnotetext{
${ }^{7}$ VRPN : http://www.cs.unc.edu/Research/vrpn/

${ }^{8}$ ARToolkit : http://www.hitl.washington.edu/artoolkit/
} 


\subsection{System initialization}

Our aim is that our telescope should be used anywhere in the world. But we had to develop a procedure to position it ideally. Our system calibration relies on an estimation of the intrinsic parameters of the pinhole camera model by simplifying the calibration pattern process. We use classical methods such as proposed in [26, 27, 28], and we adjust them to suit our environment (variable focal length of our camera). For further details about our calibration procedure one can refer to [30].

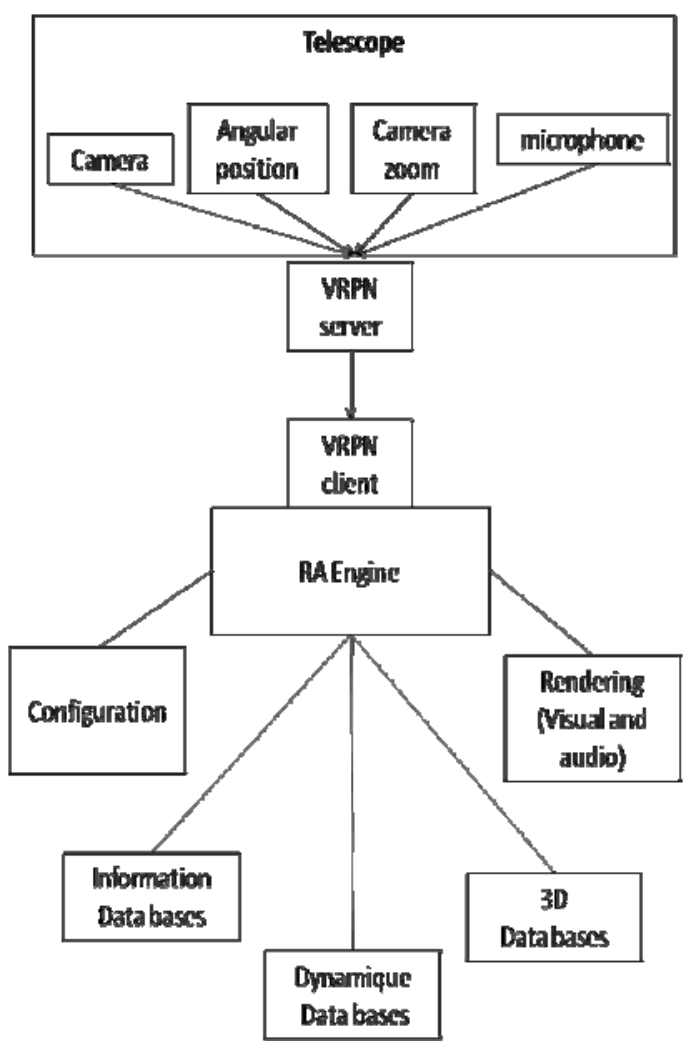

Fig. 5. Diagram of the augmented reality application.

\section{EXPERIMENTING AND USING THE TELESCOPE}

An experimentation has been set up on the "Arc de Triomphe" (Paris, France) during the festival "Futur en Seine" and presented to tourists (Fig. 6).

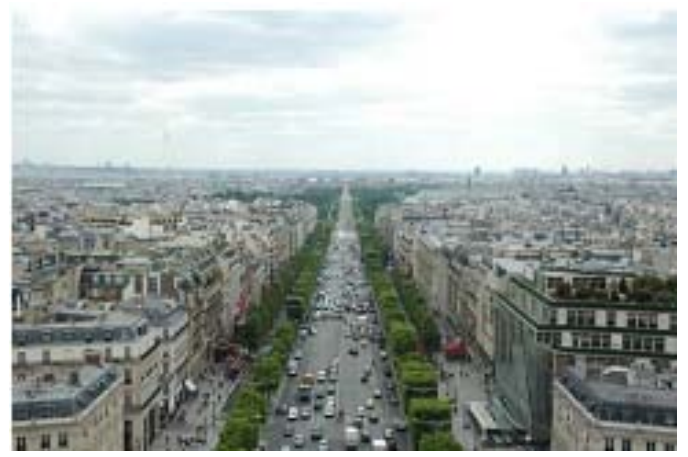

Fig. 6. View from Arc de Triomphe, Paris (France).

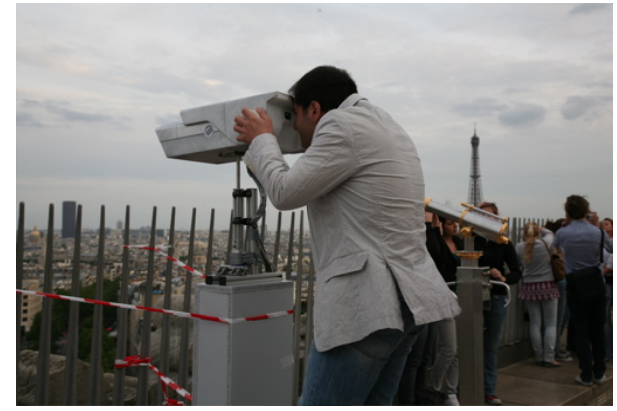

Fig. 7. A tourist looking in our Telescope (version beta).

Our telescope is linked to a computer which launches the application. We can visualize on the screen of the computer what the subjects/tourists get to see through the Telescope (Fig. 7). This application consists of displaying information balloons hovering above the historical buildings in the real landscape.

Those information balloons are more accurate when the subject/tourist zooms in the landscape with the telescope. For Example, the Fig. 8 shows pictograms in information balloons, the Fig. 9 shows information balloons with the name of the historical building. Fig. 10 shows all the information that appears progressively according the area of interest of the user.

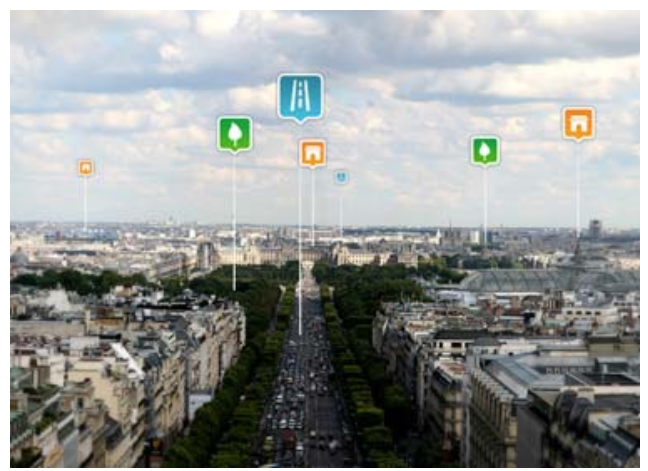

Fig. 8. View from “Arc de Triomphe, Paris (France)" with pictogram information balloon.

Some tourists have been visiting the "Arc de Triomphe" during the "Futur en Seine" on our telescope. The feedback received from the subject/tourist was excellent; many of them said that they were delighted to be allowed to test it.

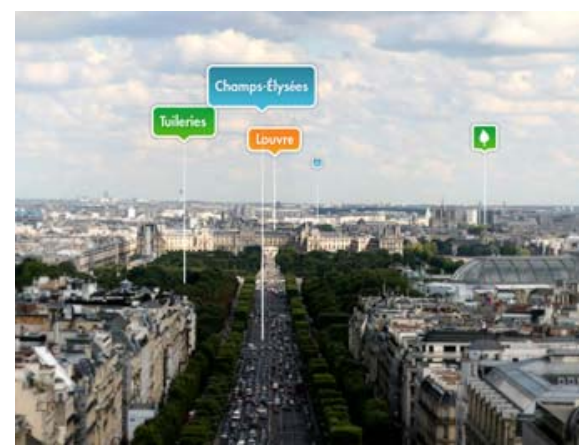

Fig. 9. View from "Arc de Triomphe, Paris (France)" with information balloon with the name of the historical building. 

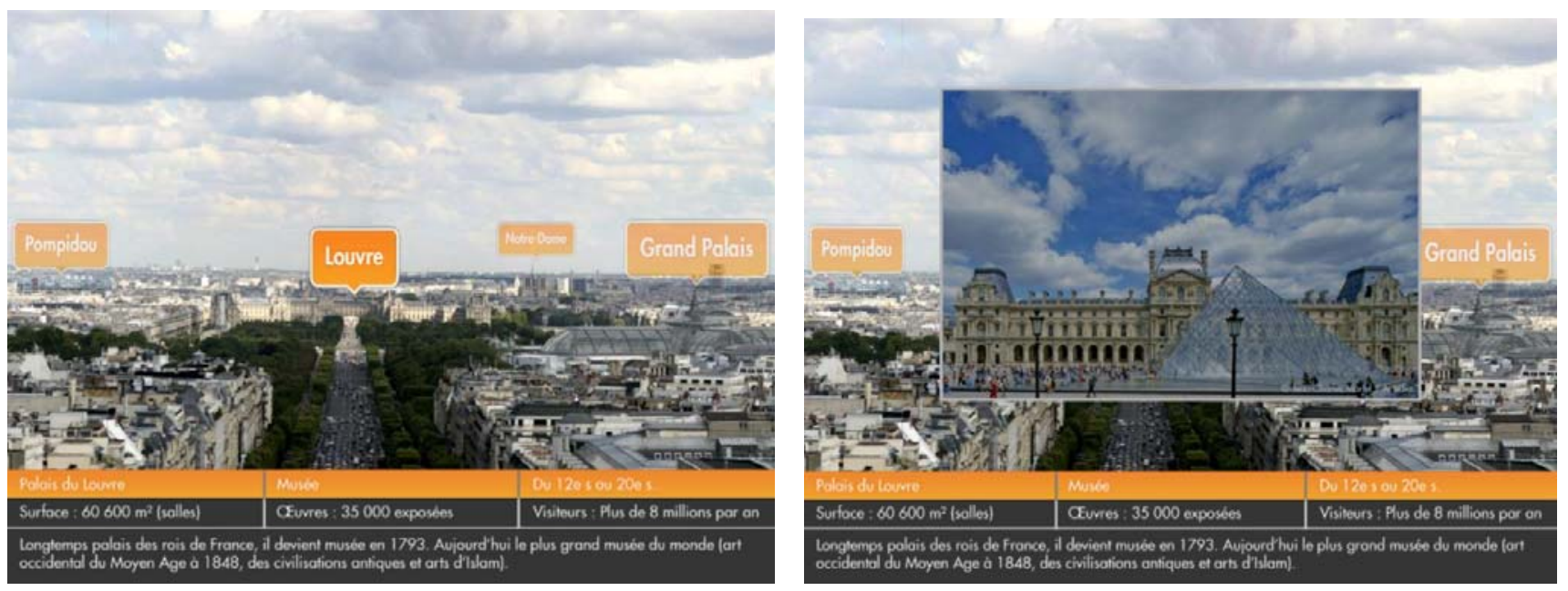

Fig. 10. (left) "Paris, Champs Elysée" avenue panoramic view with a global information related to the interest sites. (right) a focus on the "Palais du Louvre".

\section{CONCLUSION}

In this paper, we have proposed a complete study on the production of a generic augmented reality Telescope, including the underlying software, hardware, ergonomic constraints, and extensible architecture.

The platform we presented is based on dynamic calibration; it can be set up on every site around the world. The software architecture is open and portable and can be interfaced to any kind of software modeller (3DVIA, OpenSceneGraph, etc).

We also presented a final use case based on TerraNumerica cartography of Paris, and a testing protocol, together with a practical use case in the "Champs-Elysée" area. The purpose of this use case was heritage valorisation.

The System we detailed in this paper is extensible and modular. Once implemented, it can be considered as a research platform, allowing new approaches of observing and thinking the city. This System incites creativity.

\section{PERSPECTIVES}

7.1.1 Use case

We have to test the telescope on the basis of more accurate testing protocols pertaining to its functionality. The feedback we were given from the tourists was usually enthusiastic, but however we still are not aware that our telescope improves:

- Their tourism experience

- The visual search of historical building

- The identification of historical building

7.1.2 Incrustation

We could incrust several other things besides the information balloons. For example, we could incrust a 3D model of historical buildings which would temporary be hidden by fog (Fig. 11).

\subsubsection{Mutlimedia}

We wish the telescope experiences to be as interactive as can be. There is a microphone and headphone affixed to the telescope. This is particularly useful if we want sharing the experience between the users of two telescopes. For example, we could have a tourist with a telescope on the "tour Eiffel" and another on the "Arc de Triomphe”, also with a telescope. In this way they can exchange their views on the building that they both see, and even share their screen.
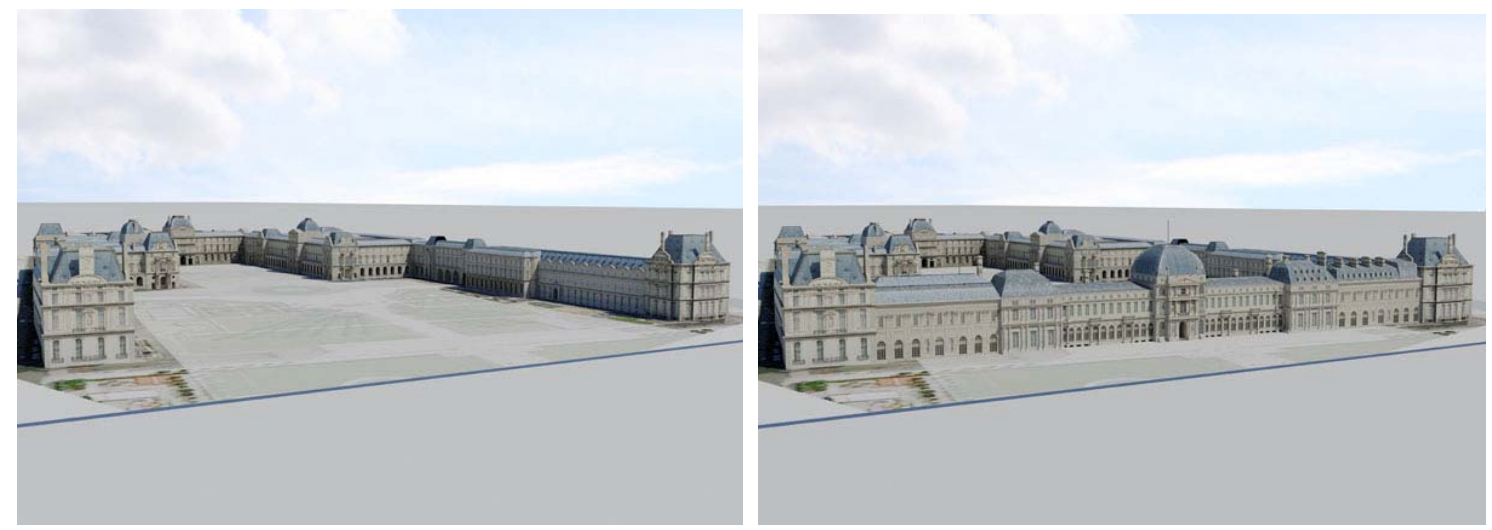

Fig. 11. Incrustation of the: (left) actual 3D model of "Palais du Louvre". (right) the 3D model of "Palais du Louvre" including the 3D reproduction of "Palais des Tuileries" (demolished in 1871). 


\section{ACKNOWLEDGMENT}

Some of the works reported in this paper were conducted and sponsored as a part of the CapDigital Business Cluster TerraNumerica $^{9}$ project and Futur en Seine ${ }^{10}$ digital world festival.

We would also like to thank:

- Thales Training and Simulation ${ }^{11}$ for the valuable information and works on the multi-focal camera calibration module.

- Centre des Monuments Nationaux ${ }^{12}$ for the production of the information databases and their valuable collaboration for conducting the experimentation from the terrace of the "Arc de Triomphe".

- Hyptique $e^{13}$ agency for the design of graphical information cartel.

We would like to thank M. Benayoun Artistic Director at the CITU ${ }^{14}$ who initiates this project.

Specials thanks go to: C. Leclercq, V. Guitteny, F. Maurel, F. Xerri, J. Prot.

\section{REFERENCES}

[1] M.A. Livingston, L.J. Rosenblum, D.G. Brown, and others, "Military applications of augmented reality". Handbook of Augmented Reality, pp. 671-706, 2011.

[2] J. Mohne, "Virtual reality for health care: a survey", In Virtual reality in neuro-psycho-physiology: cognitive, clinical and methodological issues in assessment and rehabilitation, vol. 44, 1997.

[3] J.H. Shuhaiber, "Augmented reality in surgery," in Archives of Surgery, vol. 139, pp. 170, 2004.

[4] S. Chendeb, "Chirurgie virtuelle: modélisation temps réel des tissus mous, interactions et système haptique dédié", $\mathrm{PhD}$ Thesis, Mines ParisTech Institute of Tehnology, 2007. [in french]

[5] B. Bideau, R. Kulpa, N. Vignais, and others, "Using virtual reality to analyze sports performance," in Computer Graphics and Applications, IEEE. vol. 30, pp. 14-21, 2010.

[6] R. Mintz, S. Litvak and Y. Yair, "3D-virtual reality in science education: An implication for astronomy teaching", in Journal of Computers in Mathematics and Science Teaching, vol. 20, pp. 293-305, 2001.

[7] R.T. Azuma, and others., "A survey of augmented reality", in Presence-Teleoperators and Virtual Environments, vol. 6, pp. 355385, 1997.

[8] DWF . Van Krevelen R. Poelman, "A survey of augmented reality technologies, applications and limitations", in International Journal of Virtual Reality, vol. 9, 2010.

[9] T. Ridene, "Co-registration of 3D heterogeneous geo-referenced data: contributions to the correction of mobile mapping systems surveys", PhD Thesis, Mines ParisTech Institute of Tehnology, 2010. [in french]

[10] A. Damala, I. Marchal, P. Houlier, and others, "Merging augmented reality based features in mobile multimedia museum guides", in Proceedings of the XXI International CIPA Symposium, pp. 259-264, 2007.

[11] W. Rudametkin, L. Touseau, M. Perisanidi, and others, "NFCMuseum: an Open-Source Middleware for Augmenting Museum Exhibits", in Proceedings of International Conference on Pervasive Services, pp. 6-10, 2008.

[12] S. Styliani, L. Fotis, K. Kostas, and others, "Virtual museums, a survey and some issues for consideration", in Journal of cultural Heritage, vol. 10 pp. 520-528, 2009

\footnotetext{
${ }^{9}$ TerraNumerica : http://www.terranumerica.com/

${ }^{10}$ Futur en Seine : http://www.futur-en-seine.fr/en/

${ }^{11}$ Thales : http://www.thalesgroup.com/Training_simulation.aspx

${ }^{12} \mathrm{CMN}:$ http://www.monuments-nationaux.fr/en/

${ }^{13}$ Hyptique : http://www.hyptique.fr/

${ }^{14}$ CITU : www.citu.fr
}

[13] V. Vlahakis, J. Karigiannis, M. Tsotros, and others, "Archeoguide: first results of an augmented reality, mobile computing system in cultural heritage sites", in Proceedings of Virtual reality, archeology, and cultural heritage, vol. 28 pp. 131-140, 2001.

[14] P. Dahne and J.N. Karigiannis, "Archeoguide: System architecture of a mobile outdoor augmented reality system", in Proceedings of International Symposium on Mixed and Augmented Reality, pp. 263-264, 2002.

[15] Y. Huang, Y. Liu and Y. Wang, “AR-View: An augmented reality device for digital reconstruction of Yuangmingyuan", in Proceedings Mixed and Augmented Reality-Arts, Media and Humanities, pp. 3-7, 2009.

[16] F. H. Fritz, A. Susperregui and MT Linaza, "Enhancing cultural tourism experiences with augmented reality technologies", in Proceedings 6th International Symposium on Virtual Reality, Archaeology and Cultural Heritage (VAST), 2005.

[17] E. Lange, J. L. Gill, E. Henneberry and others, "Addressing the three dimensions of sustainability in an urban river context via 3D-Visualization”, In: R. Berney (Ed.) Urban Nature. Figueroa Press, University of Southern California, Los Angeles, 35-36.

[18] D. A. Madsen and D. P. Madsen, "Engineering Drawing \& Design, Cengage Learning”, 2011, page 90.

[19] A. Lintu and M. Magnor. "Augmented Astronomical Telescop VR/AR”, pp. 203-213, Saarbuecken,Germany.

[20] A. Lințu, M. Marcus, “An Augmented Reality System for Astronomical Observations", Procedeeings of IEEE Virtual Reality 2006

[21] B. Lutz, M. Becker, D. Stricker, and others, "The augmented reality ocular", Proceedings of the 2004 ACM SIGGRAPH international conference on Virtual Reality continuum and its applications in industry, 2004, 352-354.

[22] D. Stricker, M. Zoellner, A. Bisler, and others,"Traveling in time and space with virtual and augmented reality", "Baltsavias, E. TH Zürich -ETH-:Recording, Modeling and Visualization of Cultural Heritage", Proceedings London: Taylor \& Francis, 2006, pp.431-439

[23] H. Schnädelbach, B. Koleva, M. Flintham, and others, "The Augurscope: A Mixed Reality Interface for Outdoors, CHI, changing the world, changing ourselves", minneapolis, minnesota, 2002

[24] I.P. Howard, and B.J Rogers, "Binocular Vision and Stereopsis", Oxford: Oxford psychology series, 1995.

[25] Benjamin, W. J., "Borish's Clinical refraction 2nd edition", Birmingham: Elsevier, 2007.

[26] P. S. Gravir-Imag and P. Sturm. "Self-calibration of a moving zoom-lenscamera by pre-calibration", Image and Vision Computing, pp583-589, 1997.

[27] R. Y. Tsai, "A efficient and accurate camera calibration technique for 3d machine vision", Proc. IEEE Conference on Computer Vision and Pattern Recognition, pp. 364-374, 1986.

[28] Z. Zhang. "A flexible new technique for camera calibration", Technical report, 1998.

[29] L. Leroy, P. Fuchs and G. Moreau, "Real-time adaptive blur for reducing eye strain in stereoscopic displays", TAP ACM.

[30] S. Chendeb, M. Fawaz and V. Guitteny, "Calibration of a Moving Zoom-Lens Camera for Augmented Reality Applications", ISIE 2013 [to appear] 